\title{
Transmissão de Política Macroeconômica: o caso de Brasil e Alemanha
}

\author{
Janaína Führ ${ }^{1}$
}

Maria Helena Ambrosio Dias ${ }^{2}$

Resumo: O presente trabalho estuda a transmissão macroeconômica de política fiscal entre Brasil e Alemanha no período compreendido entre o primeiro mês de 2001 até o décimo segundo mês de 2013. O objetivo é analisar os efeitos de longo prazo de transmissão da política fiscal da Alemanha sobre os agregados econômicos brasileiros: consumo, produto, e termos de troca, verificando se este efeito é beggar-thy-neighbor, beggar-thyself ou prosper-thy-neighbor. Para tanto, realiza-se uma aplicação empírica do modelo teórico de interdependência macroeconômica proposto por Corsetti e Pesenti (2001), aplicando a metodologia de séries temporais dos modelos de Vetores Autorregressivos Estruturais com variável exógena - SVARX. Os resultados obtidos através do SVARX e da análise impulso-resposta para a equação de longo prazo do produto doméstico e dos termos de troca indicam que a política fiscal alemã exerce efeito beggar-thy-neighbor como indicado no modelo teórico de Corsetti e Pesenti (2001).

Palavras-chave: Interdependência macroeconômica. Transmissão internacional de política. Modelos SVARX.

JEL: E6, E61, F4, F42 


\title{
Macroeconomic Policy Transmission: The Case Of Brazil And Germany
}

\begin{abstract}
This research studies the macroeconomic transmission of fiscal policy between Brazil and Germany in the period from the first month of 2001 to the twelfth month of 2013.The objective is to analyze the effects of long run transmission of Germany's fiscal policy on economic aggregates Brazilian: consumption, output and the terms of trade, verifying if this effect is beggar-thy-neighbor, beggar-thyselfor prosper-thy-neighbor. For this, we make an empirical application of the theoretical model of macroeconomic interdependence proposed by Corsetti and Pesenti (2001) applying the methodology of time series models Autoregressive Structural Vectors with exogenous variable - SVARX. The results from the SVARX and impulse response analysis for the long-term equation of domestic product and terms of trade indicate that the German fiscal policy has beggar-thy-neighbor effect as indicated in the theoretical model of Corsetti and Pesenti (2001).
\end{abstract}

Keywords: Macroeconomic Interdependence. International transmission of policy. SVARX models.

JEL: E6, E61, F4, F42

\section{Introdução}

Ao longo do século XX e XXI houve elevação do comércio internacional e com este o cenário macroeconômico apresentou mudanças acerca do efeito das políticas de um país sobre os demais componentes da economia mundial. Assim, conforme Cooper (1985) os países ampliaram a sua interdependência e o impacto de suas políticas macroeconômicas na economia dos demais países, além dessa interdependência ter reduzido o efeito das políticas macroeconômicas domésticas sobre a própria economia doméstica. Um exemplo desta interdependência é a magnitude da transmissão da crise econômica de 2007 que se originou nos EUA e os efeitos desta crise sobre os agregados econômicos dos países nos anos que seguiram o seu início. 
Dentre a literatura, o modelo de Corsetti e Pesenti (2001) analisa a transmissão de efeitos de política macroeconômica entre países parceiros comerciais, modelo este que serve como modelo estrutural a ser testado para a economia brasileira. A escolha da Alemanha para aplicação do modelo deve-se a sua importância no cenário econômico internacional, como integrante do G8 e como potência com forte influência na União Europeia. Conforme dados do relatório do Ministério do Desenvolvimento, Indústria e Comércio Exterior (MDIC) (2013), o Brasil foi $22^{\circ}$ importador e exportador mundial em 2012. Nesse mesmo ano, a Alemanha ocupava o $3^{\circ}$ lugar em ambos os rankings, ficando atrás apenas de China e Estados Unidos. Ainda conforme este estudo, a Alemanha foi fornecedora de 6,3\% das importações brasileiras em 2013, já no caso dos principais destinos das exportações brasileiras, a Alemanha está na $6^{\mathrm{a}}$ posição do ranking do MDIC (2013), com 2,7\% das exportações brasileiras.

Considerando a relevância da interdependência econômica e considerando o fato de haver poucos trabalhos na literatura sobre interdependência para o caso brasileiro e alemão, a presente pesquisa busca aplicar o modelo de transmissão de política macroeconômica de Corsetti e Pesenti (2001) para o caso do Brasil como país doméstico e da Alemanha como país estrangeiro. $\mathrm{O}$ objetivo geral deste trabalho é identificar se ocorre transmissão de efeitos de uma política macroeconômica entre Brasil, país doméstico, e Alemanha, país estrangeiro, no período de 2001 a 2013. Busca-se também examinar o efeito da transmissão de política fiscal entre Brasil e Alemanha, verificando se este efeito é beggar-thy-neighbor, beggar-thyself ou prosper-thy-neighbor, além de mensurar os efeitos de longo prazo de uma política fiscal alemã sobre os agregados econômicos brasileiros selecionados.

Considerando o trabalho de Dias, Dias e Punzo (2012) que tem como conclusão que uma política macroeconômica da Comunidade Europeia tem efeito prosper-thy-neighbor sobre a economia brasileira, o presente trabalho investiga para efeitos comparativos, o resultado da transmissão de política macroeconômica de um país específico da Comunidade Europeia sobre agregados econômicos brasileiros, ou seja, esta conclusão se mantém para uma das principais economias da Comunidade Europeia?

Além desta introdução, este trabalho apresenta mais 5 seções. A segunda seção contém a revisão de literatura e aplicações empíricas do modelo de Corsetti e Pesenti (2001), a terceira seção trata do referencial teórico, o modelo de Corsetti e Pesenti (2001), e a quarta seção contém a metodologia SVAR empregada na análise. A quinta seção contém os resultados da estimação das equações do modelo de Corsetti e Pesenti (2001) e a última seção contém as considerações finais. 


\section{Relações Macroeconômicas Internacionais: uma breve revisão teórica e empírica da literatura}

\subsection{Revisão teórica da literatura}

No decorrer do século XX houve ampliação do comércio internacional, maior integração financeira e aprimoramento das tecnologias referentes à comunicação. Neste contexto houve expansão da interdependência macroeconômica entre os países. Cooper (1985) sugere utilizar o termo dependência mútua no caso de dois países dependentes um do outro, e utilizar o termo interdependência para se referir ao grau de influência bidirecional de uma economia sob a outra na margem.

Alguns motivos apresentados por Cooper (1985) para a elevação da interdependência econômica derivam do progresso no transporte e na comunicação internacional, que difundem técnicas de produção e de gerência, reduzindo a dependência das exportações das adversidades localizacionais. Conforme Cooper (1985), outro fato que colaborou com a elevação da interdependência foi a redução das barreiras oficiais ao comércio, especialmente entre os países industrializados, mas estas geralmente permaneceram elevadas em países em desenvolvimento. Se a "greater familiarity with foreign financial markets, the emergence of the euro-currency market, and the lowering of barriers to capital movements have all increased the interdependence among national financial markets" " (Cooper 1985: 1207), a adoção de taxas de câmbio flexíveis introduzem barreiras modestas de incerteza de curto prazo para comércio e transações financeiras internacionais que podem provavelmente reduzir a interdependência, de acordo com o autor.

De acordo com Cooper (1969), o interesse pelos ganhos derivados da coordenação de políticas econômicas e como estes ganhos variam de acordo com o grau de interdependência econômica advêm do "great increase in international capital movements which took place after 1958, and the high sensitivity of some of these capital movements to interest rates" ${ }^{2}$ (Cooper 1969: 1).

Conforme Devereux e Wilson (1989) houve elevação da interdependência entre as economias de mercado industriais, sendo que o comércio mundial cresceu

\footnotetext{
1 Maior familiaridade com mercado financeiro estrangeiro, a emergência do euro-mercado, e a redução de barreiras ao movimento de capital elevaram a interdependência entre mercados financeiros nacionais. (Tradução da autora)

2 Crescimento significativo do movimento internacional de capitais após 1958 e da alta sensibilidade de alguns destes movimentos de capital à taxa de juros. (Tradução da autora)
} 
especialmente no início dos anos 70 e os mercados financeiros apresentaram maior grau de integração. Um argumento apresentado por Devereux e Wilson (1989) para coordenação de política macroeconômica é que no caso não cooperativo a política tem efeito spillover em outros países que pode resultar em um equilíbrio ineficiente. Mas estes ressaltam que os ganhos com a coordenação não necessariamente são significantes na prática. Outro argumento destes autores é que políticas monetárias e fiscais irregulares podem causar distorções na taxa de câmbio real e desequilíbrios comerciais, ameaçando o ambiente de negociação através do protecionismo na política.

Na evolução empírica dos benefícios da coordenação Devereux e Wilson (1989) destacam o trabalho de Oudiz e Sachs (1984) que aborda os possíveis benefícios sociais de uma coordenação internacional de políticas entre EUA, Japão e Alemanha. Dentre os modelos utilizados por estes, a coordenação de política monetária e fiscal teria benefício de no máximo metade de um por cento do PIB para os EUA, "the benefits to Germany would be about the same, and to Japan some what higher." ${ }^{3}$ (Oudiz; Sachs 1984: 5). Assim, apesar dos modelos teóricos demonstrarem possibilidade de ganhos com a coordenação, os resultados empíricos sugerem que os ganhos são pequenos e sob incerteza estes podem se reduzir, especialmente, considerando as assimetrias entre os países.

De acordo com Frankel (1988) há obstáculos para a coordenação internacional de políticas macroeconômicas, sendo que a incerteza tem efeito "on the magnitude of prospective welfare gains from international macroeconomic policy coordination." ${ }^{4}$ (Frankel 1988: 353).

A análise dos multiplicadores da política apresenta na literatura duas questões muito difundidas, "the effect on the target variables of changes in the money supply and government expenditure" 5 (Frankel 1988: 362). Conforme Frankel (1988) a primeira questão é se o fluxo de capitais que é atraído pelas taxas de juros mais elevadas é suficiente para compensar o déficit comercial resultante do aumento de renda, apreciando a moeda. Ainda conforme este, a segunda questão refere-se ao fato de que se o efeito expansionista de uma desvalorização cambial sobre a balança comercial é suficiente para compensar os efeitos contracionistas que ocorrem via renda real, preços dos insumos importados ou riqueza real de modo a aumentar a renda.

A conclusão do modelo de Mundell-Fleming de que a expansão fiscal nacional "that a domestic fiscal expansion is transmitted positively to other countries via a shift in the trade balance" ${ }^{6}$ (Frankel 1988: 364) pode ser revertida se

\footnotetext{
3 Os benefícios para a Alemanha seriam o mesmo, e para o Japão um pouco maior. (Tradução da autora) 4 Sobre a magnitude dos ganhos de bem-estar em perspectiva de coordenação internacional de políticas. (Tradução da autora)

5 O efeito sobre as variáveis de interesse de mudanças na oferta monetária e do gasto do governo. (Tradução da autora)

6 É transmitida de forma positiva para outros países por meio de uma mudança na balança comercial. (Tradução da autora)
} 
ocorrer uma resposta negativa para qualquer uma das duas perguntas citadas acima. Por exemplo, no modelo de Corsetti e Pesenti (2001), o efeito positivo da política fiscal sobre a renda para o exterior não se verifica. Já no caso de uma expansão monetária, o modelo Mundell-Fleming indica que a redução da taxa de juros gera uma saída líquida de capitais que eleva o saldo em conta corrente devido à desvalorização, considerando câmbio flutuante, tendo efeito positivo. Porém conforme Frankel (1988), os resultados empíricos indicam que a expansão monetária dos EUA agrava a sua conta corrente, resultando que a política monetária é transmitida inversamente ao caso de Mundell-Fleming, resultados consistentes com o modelo de Corsetti e Pesenti (2001) no qual uma política monetária expansionista é beggar-thyself ${ }^{7}$ no curto prazo e neutra no longo prazo.

A nova economia macroeconômica aberta (NOEM) refere se a corrente de trabalhos que buscam aprimorar o modelo Mundell-Fleming de economia aberta, mais especificamente no aspecto de interdependência macroeconômica. Neste cenário, Obstfeld e Rogoff (1995) desenvolvem uma abordagem intertemporal de transmissão de política macroeconômica internacional, pressupondo rigidez nominal de preços no curto prazo e fundamentos microeconômicos explícitos para a oferta agregada. Conforme Obstfeld e Rogoff (1995), a taxa de câmbio influência os preços da produção na moeda estrangeira, mesmo estes sendo predeterminados na moeda corrente do produtor, o que torna atrativo ao monopolista atender a variações inesperadas da demanda.

Um choque permanente de oferta monetária doméstica induz um aumento no consumo relativo doméstico, com os preços fixos no curto prazo, a depreciação da taxa de câmbio aumenta a demanda estrangeira por produtos domésticos. Este processo amplia a renda doméstica, sendo que parte desse aumento melhora a conta corrente devido a poupança dos residentes domésticos. Por outro lado, se o choque for temporário, as variações na taxa de câmbio e na conta corrente serão menores que no caso do choque permanente. Além disso, o tamanho do efeito das surpresas de política monetária sob o resultado da conta corrente depende do tamanho da economia, quanto maior a economia menor o superávit em decorrência da anulação de parte dos ganhos advindos da depreciação pelo aumento do consumo relativo.

Ainda no caso de choques monetários na estrutura de preços predeterminados a volatilidade da taxa de câmbio é reduzida em comparação com o caso de preços flexíveis. De maneira geral, os choques monetários positivos beneficiam ganhos de bem-estar, mas se houver fricção advinda do mercado de trabalho a melhora do bem-estar não seria alcançada. Porém de acordo com Dias e Dias (2010), se a distorção advém de taxação há a possibilidade de elevação dos ganhos do aumento não antecipado na demanda agregada mundial para ambos os países, sendo que o país de moeda depreciada é beneficiado pela

7 Uma política econômica beggar-thyself é aquela que quando realizada na economia do país doméstico empobrece a própria economia doméstica. 
redistribuição dos benefícios induzida por mudanças na taxa de câmbio.

No caso de uma mudança não antecipada de gastos governamentais domésticos a carga tributária doméstica adicional resultante reduz o consumo relativo e o lazer, sendo positivo o efeito líquido sobre a demanda agregada do mundo. Se o aumento dos gastos governamentais domésticos for permanente, a redução do "consumo relativo doméstico implica na redução na demanda por moeda interna e então, depreciação cambial” (DIAS; DIAS 2010: 10). Quando a mudança no gasto governamental é temporária, ocorre déficit na conta corrente deste país em consequência da redução do consumo doméstico ser menor que o aumento dos gastos governamentais domésticos. Isto conjuntamente com a depreciação cambial implica em aumento do produto doméstico relativo ao estrangeiro no curto prazo.

Betts e Devereux (2000) por sua vez, tratam da coordenação internacional de políticas monetárias num sistema de câmbio flutuante. Conforme os autores, Mundell (1968) argumenta que a política monetária é um instrumento beggar-thy-neighbor ${ }^{8}$ na presença da taxa de câmbio flexível. Ainda conforme os autores no cenário de preços rígidos na moeda do vendedor e estrutura de concorrência monopolística Obstfeld e Rogoff (1995) argumentam que uma política monetária expansionista gera efeitos spillovers positivos no bem-estar dos países que não indicam necessidade de coordenação internacional desta política, pois esta conduziria para um equilíbrio com taxa de inflação mais elevada. Para Betts e Devereux (2000) esta conclusão depende da determinação de preços, de maneira geral os autores concluem que "the degree of pricing to market determines the gains/losses from policy coordination" 9 (Betts; Devereux 2000: 740).

Dentre a literatura de transmissão internacional de política macroeconômica o presente trabalho utiliza o modelo de interdependência macroeconômica desenvolvido por Corsetti e Pesenti (2001), como a fundamentação teórica, sendo este desenvolvido na seção 3. Na seção a seguir são apresentados alguns trabalhos recentes dentre essa análise.

\subsection{Evidências empíricas sobre transmissão macroeconô- mica internacional}

Nesta seção são apresentados alguns trabalhos recentes sobre interdependência macroeconômica que desenvolvem principalmente aplicações do modelo de Corsetti e Pesenti (2001). Dentro da literatura de transmissão macroeconômica Dias e Dias (2010) examinam o caso de Brasil e Argentina como país doméstico e Estados Unidos como país estrangeiro para o período

8 A política econômica é considerada beggar-thy-neighbor quando a implementação desta empobrece a economia do país parceiro comercial.

9 O grau de precificação a mercado determina os ganhos/perdas da coordenação de política. (Tradução da autora) 
de 1980 a 2009 empregando o modelo SVAR. A análise empírica utiliza o desvio em relação à tendência de longo prazo das séries, aplicando o filtro Hodrick Prescott (HP). Uma alteração da política de gastos americana que supere os gastos relativos do Brasil em proporção dos respectivos PIB altera os termos de troca, depreciando os termos de troca domésticos, o que melhora o balanço de pagamentos. Porém, no longo prazo a diminuição dos encaixes reais domésticos reduz o poder de compra das famílias reduzindo a taxa de crescimento do PIB e do consumo, confirmando o efeito beggar-thy-neighbor.

Ainda considerando Brasil e Argentina como país doméstico Dias, Dias e Punzo (2012) estimam o modelo SVAR para Europa como país estrangeiro, verificando o impacto da política fiscal da comunidade europeia sobre o consumo e o produto brasileiro considerando mudanças de longo prazo nos gastos governamentais da Comunidade Europeia para o período de 1980-2009. De maneira geral, considerando o desvio em relação à tendência de longo prazo, uma política fiscal expansionista europeia, seja permanente ou transitória, causa perda de bem-estar na Argentina no curto e no longo prazo. Já no caso do Brasil ocorrem ganhos de bem-estar no curto e no longo prazo através de incrementos no consumo e no produto se a política for permanente, mas não afeta os preços domésticos, assim a política fiscal da Europa é prosper-thy-neighbor ${ }^{10}$. Se a política fiscal europeia for temporária esta causa um movimento cíclico no consumo e produto do Brasil, mas não altera bem-estar.

Considerando a escassez de aplicações do modelo de transmissão e interdependência de política de Corsetti e Pesenti (2001) para o caso do Brasil e da Alemanha e destacando o caso de efeito prosper-thy-neighbor entre Brasil e Europa citado aqui, este trabalho analisa o caso de Brasil e Alemanha para verificar se este efeito se aplica apenas para a Europa como um todo ou se aplica também para um país em particular da Europa.

\section{O Modelo Teórico de Transmissão Macroeconômica Internacional}

\subsection{O modelo de Corsetti e Pesenti (2001) de interdepen- dência macroeconômica}

De acordo com Corsetti e Pesenti (2001), a nova economia macroeconômica aberta (new-open-economy-macroeconomics) destaca as inter-relações entre fontes externas e internas de distorções econômicas. As distorções econômicas podem ser associadas com o grau de abertura de uma economia ao comércio, por meio do poder de um país afetar seus termos de troca, manipulando a 
oferta de produtos.

Corsetti e Pesenti (2001) apresentam um modelo de equilíbrio geral de dois países com rigidezes nominais, em que as firmas agem competitivamente, mas o mercado de trabalho e de bens não são perfeitamente competitivos. O modelo introduz rigidez nominal no curto prazo, salários nominais são predeterminados com a antecipação de um período, sendo que na ausência de choques estes se ajustam ao seu nível de equilíbrio de estado estável. A rigidez de preços advém de fricções reais no mercado de trabalho, seja em decorrência da presença de sindicatos ou de qualificação específica. No entanto, o grau de competição monopolística é determinado pela tecnologia (Dias e Dias 2010: 15).

A estrutura do modelo de Corsetti e Pesenti (2001) inclui duas economias, doméstica e estrangeira, a função utilidade especificada para cada agente $\mathbf{j}$ que no agregado formará um continuum de agentes econômicos.

\begin{abstract}
"Suas decisões dependem do comportamento a respeito de um índice de consumo para uma quantidade desejada de bens domésticos e estrangeiros; uma quantidade desejada de saldos monetários reais; da utilidade dos bens públicos G; e a quantidade de trabalho (l) ofertada pelo agente j. A função utilidade do agente j conta com uma taxa de desconto no tempo $\beta$, igual a [1/ $(1+\delta)]$. Enquanto, $1 / \rho$ é a elasticidade-substituição intertemporal entre consumo e lazer" (Dias; Dias 2010: 15).
\end{abstract}

As preferências para bens de consumo são simétricas tanto dentro quanto entre as nações, sendo assim os índices de preços são idênticos em ambos os países. A função de produção de cada economia é descrita por uma relação CES entre tecnologia e um continuum de serviços de trabalho diferenciados utilizados na produção de Y. Destaque que os termos de troca são sensíveis a um impulso de choques de política na economia mundial.

Na restrição orçamentária individual em ambos os países os agentes mantêm dois tipos de ativos, moeda nacional e título internacional B, sendo que em nível de mercado mundial o título B tem posição de equilíbrio no curto e no longo prazo devido a oferta líquida igual a zero desse título. Isso tem como implicação o fato que "in equilibrium, the ratio of Home to Foreign consumption is Constant at $\gamma / 1-\gamma$ : the mechanism of adjustment to shocks in the world economy hinges exclusively upon variations of the terms of trade, without changes in national net-asset positions" ${ }^{11}$ (Corsetti; Pesenti 2001:

11 Em equilíbrio, a razão de consumo doméstico para estrangeiro é constante em $\gamma / 1-\gamma$ : o mecanismo de ajustamento a choques na economia mundial depende exclusivamente sobre variações dos termos de troca, sem mudanças nas posições líquidas de ativos nacionais. (Tradução da autora) 
432). Sendo que o quadro 1 com as soluções do modelo de Corsetti e Pesenti (2001) encontra-se no apêndice.

\subsection{Implicações do modelo de Corsetti e Pesenti (2001) para política econômica}

O modelo de Corsetti e Pesenti (2001) destaca que os efeitos de choques de política econômica sobre o produto do país estão sujeitos ao grau de abertura do país e de seu tamanho frente ao mercado mundial. No caso de uma economia fechada uma pequena expansão monetária permanente não antecipada eleva o bem-estar se o maior nível de consumo doméstico for superior a "desutilidade" do trabalho.

Já no caso de uma economia pequena e aberta uma política monetária expansionista elevaria a oferta mundial de moeda, reduzindo a taxa real de juros, resultando em fuga de capitais da economia do país que implementou a expansão monetária, depreciando a taxa de câmbio. Assim ocorreria a elevação da demanda agregada e a redução do poder de compra da moeda doméstica, sendo que as externalidades negativas da redução do poder de compra podem ser superiores as externalidades positivas da demanda. De acordo com Dias e Dias (2010), no longo prazo a política monetária seria neutra em decorrência dos preços acompanharem o movimento da oferta monetária, gerando o retorno ao patamar inicial do produto, do consumo, da taxa de câmbio e dos saldos monetários reais.

Considerando uma economia grande e aberta os efeitos da política monetária expansionista seriam acentuados em comparação a economia pequena, sendo que ambos os casos essa política pode ser beggar-thyself. Corsetti e Pesenti (2001) destacam que a expansão monetária implementada conjuntamente poderia retirar o efeito da taxa de câmbio, beneficiando os países em direção ao seu produto potencial.

Uma política fiscal expansionista por sua vez pode apresentar efeito beggar-thy-neighbor, sendo os choques fiscais descritos como mudanças inesperadas no longo prazo (acima de um período) na razão entre produto e produto líquido dos gastos governamentais, pressupondo que os gastos governamentais incidem apenas sobre os produtos domésticos. Além disso, os choques fiscais permanentes no modelo têm como consequência que o nível de gastos de curto prazo seja igual ao nível de longo prazo. Segundo o modelo, uma política fiscal não antecipada no curto prazo não impacta na demanda doméstica e no emprego, para um dado patamar dos termos de troca. 
Portanto a política fiscal temporária é neutra, não afetaria a economia por mais de um período, mas um choque permanente dos gastos do governo não é neutro. Os efeitos da política fiscal permanente dependem do parâmetro $\rho$ do modelo, que representa o inverso da elasticidade-substituição intertemporal $(1 / \rho)$. De acordo com Corsetti e Pesenti (2001), no caso de choques fiscais permanentes e que resultem em aumento da demanda por bens domésticos um ajustamento para cima dos salários domésticos de longo prazo será requerida, resultando em elevação dos preços relativos domésticos e apreciação da moeda em termos reais. Em decorrência do ajustamento dos salários reais o crescimento do produto seria em menor proporção que o crescimento dos gastos governamentais, o consumo mundial reduziria e os preços elevariam em ambos os países. Além disso, a economia deslocaria se para um novo equilíbrio com um nível mais elevado do produto e com consumo reduzido em relação ao estado inicial.

No caso uma política fiscal expansionista no país estrangeiro, o gasto governamental mundial $\left(\mathrm{g}_{\mathrm{w}}\right)$ aumentaria, impactando na economia doméstica no longo prazo. Inicialmente essa expansão fiscal aumentaria a demanda por bens estrangeiros, a qual poderia ser atendida se o salário real do país estrangeiro aumentar. Consequentemente elevaria o preço do produto estrangeiro, deteriorando os termos de troca domésticos e reduziria o salário real doméstico. Assim, o consumo privado estrangeiro se reduz devido ao crescimento do produto estrangeiro ocorrer em menor proporção que a elevação dos gastos governamentais estrangeiros. O consumo privado doméstico por sua vez reduziria em decorrência da deterioração dos termos de troca nacionais, essa redução pode ser ampliada se a inflação doméstica elevaria o que reduz os saldos monetários reais.

O efeito de uma expansão fiscal no país estrangeiro sobre o produto doméstico depende de $\rho$. "Since the Foreign fiscal shock reduces the availability of Foreign goods to world consumers ( $\mathrm{Y}^{*}-\mathrm{G}^{*}$ falls), world demand for Home goods increases if the two national goods are substitutes $(\mathrm{p}>1)$, and falls otherwise" ${ }_{12}$ (Corsetti; Pesenti 2001: 440). Na transmissão intertemporal de políticas econômicas o modelo de Corsetti e Pesenti (2001) indica que os efeitos spillovers de uma expansão fiscal permanente podem ser beggar-thy-neighbor.

\section{Especificação do Modelo Svar}

O método empírico empregado para desenvolver a análise é o modelo SVAR

12 Como o choque fiscal estrangeiro reduz a disponibilidade de bens estrangeiros ( $\mathrm{Y}^{*}-\mathrm{G}^{*}$ cai), a demanda mundial por bens domésticos aumenta se os bens doméstico e estrangeiro forem substitutos, $(\rho>1)$, e reduz caso contrário. (Tradução da autora) 
(Structural Vector Autoregressive) com uma variável exógena ou SVARX, pois permite avaliar os choques temporários ou de curto prazo e estimar os efeitos de longo prazo ou choques permanentes de políticas econômicas de um país sobre a economia do outro país, além de considerar a existência de choques estruturais. Conforme ressaltado por Dias e Dias (2010), os modelos SVAR permitem avaliar a interação entre política fiscal e monetária, na relação entre encaixes reais e política fiscal, de modo a interferir na eficiência da política monetária em afetar moeda real.

A especificação do modelo SVAR segue a apresentação formal de Dias e Dias (2010) sobre os modelos SVAR. Conforme estes, a base destes modelos é a relação entre previsões de erros e inovações estruturais. Os autores seguem a apresentação proposta por Hamilton (1994).

As restrições impostas pelo sistema são de duas ordens: i) ordem das equações; e ii) a exogeneidade contemporânea das variáveis. Ambas determinam a disposição e as restrições dos coeficientes da matriz. Além disso, ambas as restrições devem ser derivadas do modelo teórico que esteja sendo testado empiricamente.

Sendo que a seguir será estimado conforme o modelo de Corsetti e Pesenti (2001) o impacto de longo prazo da política fiscal alemã sobre os agregados macroeconômicos brasileiros produto e termos de troca.

\section{Modelo Empírico e Resultados Econométricos}

As equações do modelo de Corsetti e Pesenti (2001) utilizadas neste trabalho referem-se às equações de longo prazo do quadro 1 do apêndice, referentes ao produto doméstico e os termos de troca. Para a aplicação do SVAR com variável exógena (SVARX) ao modelo de Corsetti e Pesenti (2001) as variáveis foram log-linearizadas, sendo que segue abaixo a listagem das variáveis empregadas no modelo, sendo que esta nomenclatura será utilizada nos testes. Estas variáveis foram obtidas junto ao DESTATIS, EUROSTAT, IBGE e IPEADATA.

- dummy_crise: dummy referente a crise internacional de 2008 (m8 2008 - m11 2009);

- lncambio: log da taxa de câmbio real efetiva;

- $\operatorname{lng}_{\mathrm{a} a}: \log$ do gasto governamental estrangeiro; 
- $\quad \operatorname{lng}_{\mathrm{br}}: \log$ do gasto governamental doméstico;

- $\operatorname{lng}_{\mathrm{r}}: \log$ do gasto relativo;

- $\quad \operatorname{lng}_{\mathrm{w}}: \log$ do gasto governamental mundial, que é composto pelo $\operatorname{lng}_{\mathrm{br}}$ e pelo $\operatorname{lng}_{\mathrm{al}}$;

- lnpib_br: log do PIB doméstico.

Antes da estimação das equações foi verificado o comportamento estacionário das séries, sendo realizados os testes de raiz unitária Dickey Fuller (DF), Phillips-Perron (PP), KPSS (Kwiatkowski, Phillips, Schmidt, and Shin) e Dickey Fuller Ampliado (DF-GLS). O resultado encontra se na tabela 1 do anexo. As variáveis lncambio e lnpib_br foram empregadas em primeira diferença.

\subsection{Impactos de uma política fiscal da Alemanha no pro- duto de longo prazo}

A equação 5 do quadro 1 representa o impacto de uma expansão da política fiscal internacional, sobre o produto brasileiro de longo prazo. Antes de estimar o modelo SVARX entre $\operatorname{lng}_{\mathrm{w}}$ e $\operatorname{lng}_{\mathrm{br}}$ e dlnpib_br com a variável exógena dummy_crise, testou-se o número de defasagens ótimas, nesse caso o resultado é quatro defasagens conforme os critérios LR, FPE, AIC e HQIC.

Posteriormente, foi regredido o modelo VARX base com quatro defasagens, os lags utilizados foram 1, 2, 4 e 5, os resultados mostram como significativo o coeficiente da variável dlnpib_br para o lag $1(p$-value $=0,066)$ e o coeficiente das variáveis $\operatorname{lng}_{\mathrm{br}}$ e $\operatorname{lng}_{\mathrm{w}}$ para os lags 2, 4 e 5 ( $p$-value = 0,063; 0,000 e 0,009; 0,028; 0,000 e 0,002 respectivamente) na equação do produto a um nível de significância de 1\% enquanto que a dummy_crise mostrou-se não significativa. Para confirmar esse diagnóstico após a regressão foi realizado o teste do Multiplicador de Lagrange (LM) e o teste de Wald de exclusão de defasagens, sendo que a partir do primeiro teste pode se rejeitar $\mathrm{H}_{\mathrm{o}} \operatorname{com} p$-value $=$ 0,03753 . A partir do segundo teste os resultados sugerem que as defasagens 2, 4 e 5 não devem ser excluídas do modelo ( $p$-value = 0,007; 0,000 e o,00o respectivamente), assim optamos por manter quatro como o número de lags.

Logo, foi realizado o teste de causalidade de Granger com a hipótese nula que $\operatorname{lng}_{\mathrm{w}}$ não causa no sentido de Granger dlnpib_br, o resultado indica com um $\chi^{2}=18,543$ e um $p$-value $=0,001$ que $\mathrm{H}_{\mathrm{o}}$ pode ser rejeitado a qualquer nível de significância, e, portanto a probabilidade de $\operatorname{lng}_{\mathrm{w}}$ causar dlnpib_br é significativa no curto prazo, uma vez condicionada a um número de quatro lags.

O teste de normalidade dos resíduos Jarque-Bera forneceu um $\chi^{2}=318,347$ 
e um $p$-value $=0,000$ que permitem rejeitar a hipótese nula que os resíduos seguem uma distribuição normal como o confirma o Gráfico $3{ }^{13}$. Porém, para determinar se a variância e a covariância dos mesmos são estacionárias, foram realizados os testes de estacionariedade, cujos resultados confirmam que embora os resíduos não estejam normalmente distribuídos são estacionários.

O teste de estabilidade do VARX confirmou sua estabilidade, todos os Eigenvalues da matriz de coeficientes foram menores que um. Assim, foi estimado o modelo SVARX de longo prazo com o número de defasagens recomendado pelos testes acima. Os resultados desta equação estão a seguir.

$$
\begin{aligned}
& \mathrm{y}_{\mathrm{t}}=\left(\ln g_{\mathrm{br}}, \mathrm{d} \ln \mathrm{pib} \_\mathrm{br}\right)=\left[\begin{array}{l}
\operatorname{lng}_{\mathrm{w}} \\
\operatorname{lng}_{\mathrm{br}} \\
\mathrm{d} \ln \mathrm{pib}_{-} \mathrm{br}
\end{array}\right] \mathrm{C}\left(\operatorname{lng} \mathrm{g}_{\mathrm{br}}, \mathrm{d} \ln \text { pib_br }\right)= \\
& {\left[\begin{array}{l}
2,3116 \\
(0,1335)^{*}
\end{array}\right.} \\
& \text { O } \\
& 7,2027 \quad 1,3907 \\
& \begin{array}{l}
(0,4311)^{*} \\
-0,0156 \\
(0,044)^{*}
\end{array} \\
& (0,0803)^{*} \\
& -0,0083 \\
& 0,0571 \\
& e_{t}\left(\operatorname{lng}_{b r}, d \ln p i b_{-} b r\right)=\left[\begin{array}{c}
p_{t} \\
g_{t} \\
t_{t}
\end{array}\right] \\
& \left.(0,0033)^{*}\right]
\end{aligned}
$$

O resultado do modelo SVARX indica que a relação de longo prazo entre a política fiscal internacional e a taxa de crescimento do produto doméstico ${ }^{14}$ proposta pela teoria se confirma, dado que todos os coeficientes são significativos a 1\%. Dessa forma o modelo estimado foi utilizado para realizar dois choques, observados dentro do período de 18 meses. O primeiro choque representa um impulso único na variável $\operatorname{lng}_{\mathrm{w}}$ sobre a variável dlnpib_br, que corresponde ao lado esquerdo do gráfico 1 . O segundo apresenta um impulso acumulado na variável $\operatorname{lng}_{\mathrm{w}}$ e a resposta da variável dlnpib_br, este é apresentado no lado direito do gráfico 1.

Um impulso único, que representa um choque de política fiscal em que os gastos do governo da Alemanha superem proporcionalmente os do Brasil apenas uma vez, portanto temporário, faz com que a taxa de crescimento

13 Os resultados dos testes de normalidade e estacionariedade dos resíduos são apresentados no gráfico 3 e na tabela 2 dos Anexos.

14 dln corresponde a aproximação da taxa de crescimento, sendo que de agora em diante será empregado o termo taxa de crescimento. 
do produto brasileiro seja reduzida em até $0,016 \%$ por um curto período de tempo. Mas a tendência da taxa de crescimento do produto doméstico é retornar à estabilidade, ou seja, oscilar em torno da taxa de crescimento de longo prazo. De maneira geral, choques fiscais positivos aumentam a variância do produto inicialmente, mas não afeta o produto de maneira permanente.

Se a expansão da política fiscal alemã for acumulativa em 1 desvio-padrão da variável $\operatorname{lng}_{\mathrm{w}}$, ou seja, uma política permanente de aumento dos gastos na Alemanha proporcionalmente maiores que os do Brasil levariam a uma redução permanente na taxa de crescimento do consumo no longo prazo. Esta redução pode chegar a $0,016 \%$, mas a queda na taxa de crescimento do produto reduz-se ao longo do tempo. Assim, a política fiscal alemã apresenta efeito beggar-thy-neighbor, conforme resultado do modelo teórico do modelo de Corsetti e Pesenti (2001).

GRÁFICO 1 - IMPULSO-RESPOSTA: IMPULSO ( LNG $_{\mathrm{w}}$ ) RESPOSTA (DLNPIB_ $\mathrm{BR})$
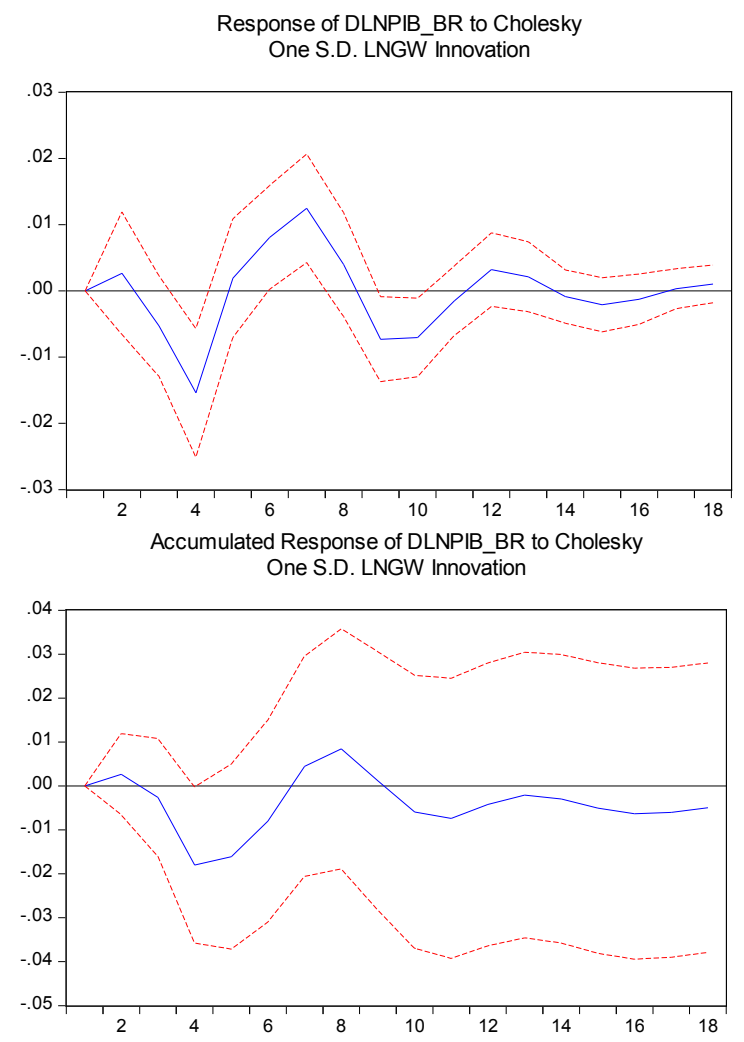

Fonte: Resultados da pesquisa no Eviews. 
Conforme o modelo teórico de Corsetti e Pesenti (2001) a transmissão da política fiscal do país estrangeiro ocorreria de maneira negativa, causando um efeito beggar-thy-neighbor. Esta transmissão está associada ao impacto da depreciação dos termos de troca brasileiros, via elevação dos preços domésticos devido à elevação do preço dos bens transacionáveis. Uma explicação para as externalidades negativas do aumento dos gastos da Alemanha sobre a economia brasileira pode estar relacionada à depreciação dos termos de troca. Considerando que as exportações brasileiras para a Alemanha são concentradas em produtos de fator agregado básico e as importações brasileiras advindas da Alemanha são concentradas em produtos manufaturados, conforme dados do MDIC (2014), o aumento da demanda mundial advinda da política fiscal expansionista da Alemanha causa um aumento dos preços relativos dos bens transacionáveis e causa uma queda no valor internacional atribuído aos bens brasileiros, afetando em última instância o produto interno.

Considerando que o valor das exportações brasileiras para a Alemanha é inferior ao valor das importações, a balança comercial Brasil-Alemanha é deficitária em todo o período de análise e considerando que teoricamente o consumo das famílias brasileiras iria ser reduzido, a taxa de crescimento do produto de longo prazo da economia brasileira seria reduzida. Assim, a elevação dos gastos governamentais alemães teria um efeito beggar-thy-neighbor sobre a taxa de crescimento do produto brasileiro de longo prazo confirmado pelo modelo empírico.

\subsection{Impactos de uma política fiscal da Alemanha nos ter- mos de troca de longo prazo}

A seguir analisa-se o impacto de uma expansão da política fiscal internacional, sobre os termos de troca de longo prazo, onde os termos de troca referem-se à taxa de câmbio efetiva real brasileira em relação aos 16 maiores parceiros comerciais do Brasil, entre os quais encontra-se a Alemanha. Antes de estimar o modelo SVARX entre lng e dlncambio com a variável exógena dummy_crise, testou-se o número de defasagens ótimas, nesse caso o resultado segundo os critérios de LR, FPE, AIC, HQIC e SBIC indicaram como número de defasagens ótimas duas defasagens.

Posteriormente, foi regredido o modelo VARX base com duas defasagens, os lags utilizados foram 1 e 8 , os resultados mostram como significativo o coeficiente da variável dlncambio para o lag 1 ( $p$-value $=0,000$ ) e o coeficiente da variável $\operatorname{lng}_{\mathrm{r}}$ para o lags $8(p$-value $=0,042)$ na equação dos termos de troca a 
um nível de significância de 1\% enquanto que a dummy_crise mostrou-se não significativa. Para confirmar esse diagnóstico após a regressão foi realizado o teste do Multiplicador de Lagrange (LM) e o teste de Wald de exclusão de defasagens, a partir do primeiro teste obtém-se um $p$-value $=0,43191$, não se pode rejeitar $\mathrm{H}_{\mathrm{o}}$ A partir do segundo teste os resultados sugerem que as defasagens 1 e 8 não devem ser excluídas do modelo, $p$-value = 0,000 e 0,045 respectivamente.

Novamente, o teste de causalidade de Granger com a hipótese nula que $\operatorname{lng}_{\mathrm{r}}$ não causa no sentido de Granger dlncambio, $o$ resultado indica com um $\chi^{2}=$ 4,6811 e um $p$-value $=0,0096$ que $\mathrm{H}_{0}$ pode ser rejeitado a qualquer nível de significância, e, portanto a probabilidade de $\operatorname{lng}_{\mathrm{r}}$ causar dlncambio é significativa no curto prazo, uma vez condicionada a um número de dois lags. $\mathrm{O}$ teste de normalidade dos resíduos Jarque-Bera forneceu um $\chi^{2}=176,920$ e um $p$-value $=0,000$ que permitem rejeitar a hipótese nula que os resíduos seguem uma distribuição normal como o confirma o Gráfico $3^{15}$. Porém, a variância e a covariância podem ser estacionárias. Para confirmar isso foram feitos os testes de estacionariedade, os resultados confirmam que embora os resíduos não estejam normalmente distribuídos são estacionários.

O teste de estabilidade do VARX confirmou sua estabilidade, todos os Eigenvalues da matriz de coeficientes foram menores que um. Com base no número de defasagens recomendados pelos testes, foi estimado o modelo empírico equivalente à equação de longo prazo dos termos de troca. Os resultados são apresentados na equação (2), sendo que todos os coeficientes são significativos a $1 \%$, indicando que os termos de troca são influenciados diretamente pela política fiscal da Alemanha relativa a política fiscal da Brasil e que estes são o canal de transmissão da política fiscal alemã para os agregados econômicos brasileiros.

$$
\begin{aligned}
& \mathrm{y}_{\mathrm{t}}=(\mathrm{d} \text { lncambio })=\left[\begin{array}{c}
\operatorname{lng}_{\mathrm{r}} \\
\mathrm{d} \ln \text { cambio }
\end{array}\right] \mathrm{C}(\mathrm{d} \text { ln cambio })=\left[\begin{array}{ll}
1.2647 & 0 \\
(0,073)^{*} & \\
0,0206 & 0,0439 \\
(0,0038)^{*} & (0,0026)^{*}
\end{array}\right] \mathrm{e}_{\mathrm{t}} \\
& (\text { d ln cambio })=\left[\begin{array}{c}
\mathrm{p}_{\mathrm{t}} \\
\mathrm{t}_{\mathrm{t}}
\end{array}\right]
\end{aligned}
$$

No gráfico 2 são apresentados o impulso-resposta único e acumulado para um período de 18 meses. Os resultados mostram que os impactos de um choque único primeiramente são negativos, após o $8^{\circ}$ mês estes se tornam positivos, 
criando um ciclo. Enquanto que os choques acumulados no tamanho de 1 desvio-padrão da variável $\operatorname{lng}_{\mathrm{r}}$ iniciam um processo de depreciação dos termos de troca brasileiros no longo prazo. Quando ocorre uma variação permanente na política fiscal alemã relativa esta afeta a variação dos termos de troca em até $0,021 \%$ no longo prazo, sendo que primeiramente o efeito é negativo e após o $8^{\circ}$ mês é positivo. Assim, o efeito é beggar-thy-neighbor como ocorre no caso do produto e no caso dos saldos monetários reais.

\section{GRÁFICO 2 - IMPULSO-RESPOSTA: IMPULSO (LNG ${ }_{\mathrm{R}}$ ) RESPOSTA(DLNCAMBIO)}

Response of DLNCAMBIO to Cholesky

One S.D. LNGR Innovation
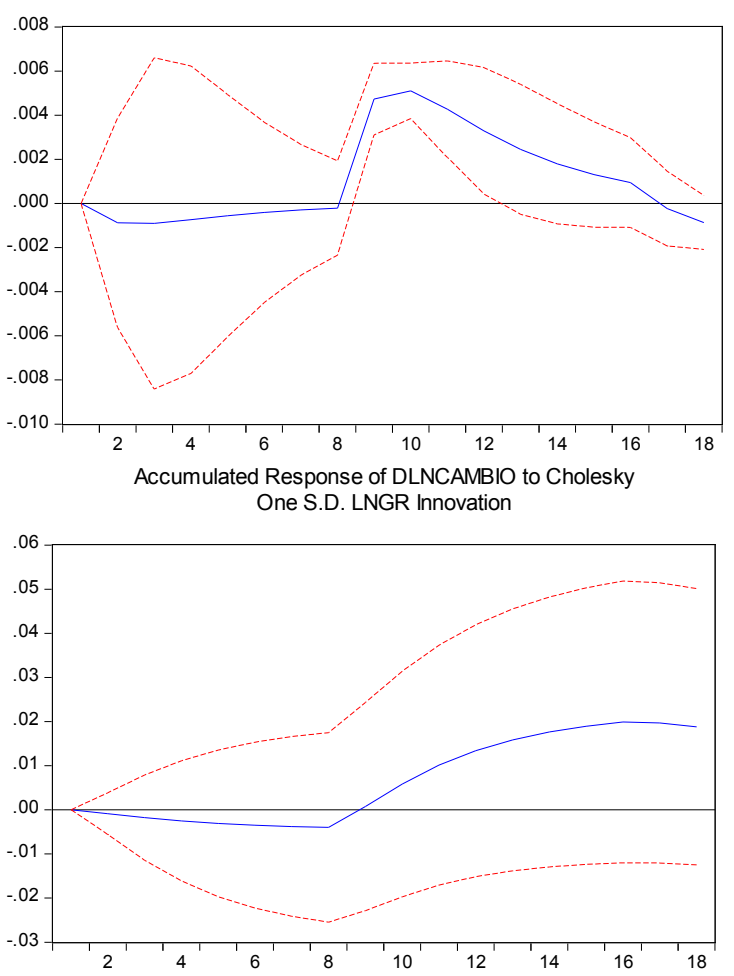

Fonte: Resultados da pesquisa no Eviews.

No modelo teórico, a depreciação dos termos de troca brasileiros frente a um aumento do gasto governamental da Alemanha resultaria em uma maior competitividade inicial dos produtos domésticos transacionados com a Alemanha. Esta poderia melhorar o resultado da balança comercial brasileira afetando positivamente a economia brasileira. Porém, os efeitos cumulativos 
indicam que, inicialmente, poderia haver efeitos negativos no longo prazo devido à elevação dos preços domésticos e causar perda de poder de compra da moeda doméstica num primeiro momento. No entanto, estes efeitos podem estar sendo contrabalanceados pelas políticas de estímulo ao consumo brasileiro no curto prazo, destacados por Contri (2010), efeitos esses que não permanecem no longo prazo. Assim, os resultados do modelo empírico estão de acordo com os esperados teoricamente, efeito beggar-thy-neighbor da política fiscal alemã sobre os termos de troca brasileiros.

\section{Considerações Finais}

Com a intensificação da globalização dos últimos 20 anos, o avanço das tecnologias de comunicação digital, a maior integração do mercado financeiro e o crescimento do comércio internacional, as políticas econômicas de um país afetam outros países de maneira direta e/ou indireta com maior impacto. Assim, adquiriram destaque às questões referentes à ampliação da interdependência entre os países, ao impacto das políticas macroeconômicas domésticas na economia dos demais países e aos canais de transmissão internacional da política macroeconômica.

Neste trabalho foi empregado o modelo de Corsetti e Pesenti (2001) de transmissão de política macroeconômica para o caso de Brasil como economia doméstica e Alemanha como economia estrangeira. Os resultados obtidos através do SVARX e da análise impulso-resposta para a equação de longo prazo do produto doméstico e dos termos de troca indicam que a política fiscal alemã exerce efeito beggar-thy-neighbor como indicado no modelo teórico de Corsetti e Pesenti (2001).

Estes resultados não corroboram com o efeito encontrado por Dias, Dias e Punzo (2012) indicando que este não é específico para a Alemanha, podendo estar sendo influenciado pela heterogeneidade dos países da Comunidade europeia. Esse resultado também pode ser influenciado pelo fato de que a análise de Dias, Dias e Punzo (2012) emprega na análise econométrica as séries com base nos desvios em relação às respectivas tendências de longo prazo das séries, inclusive das variáveis fiscais. 


\section{Referências Bibliográficas}

Betts, C.; Devereux, M. B. (2000). "International Monetary Policy Coordination and Competitive Depreciation: A Reevaluation". Journal of Money, Credit and Banking, v. 32(4), pp. 722-745.

Contri, A. L.(2010). "A retomada do crescimento brasileiro em 2010 e a política econômica”. Indicadores Econômicos FEE, Porto Alegre, v. 38(2),pp. 17-26.

Cooper, R. N. (1985). "Economic Interdependence and Coordenation of Economic Policies”. Handbook of Internacional Economics, v. 2, pp. 1195-1234.

Cooper, R. N. (1969). "Macroeconomic Policy Adjustment in Interdependence Economies". The Quarterly Journal of economics, v. 83(1), pp. 1-24.

Corsetti, G; Pesenti, P. (2001). "Welfare and Macroeconomic Interdependence". The Quarterly Journal of Economics, v. 116(2), pp. 421-445.

Destatis. Lange Reihen ab 1970. Disponível em: < https://www.destatis.de/DE/ ZahlenFakten/GesamtwirtschaftUmwelt/VGR/VolkswirtschaftlicheGesamtrechnungen.html\#Tabellen > Acesso em: jul. 2014.

Devereux, M.; Wilson, T. A. (1989). "International Co-ordination of Macroeconomic Policies: a Review”. Canadian Public Policy/Analyse de Politiques, v. 15, pp. S2O-S34.

Dias, M. H. A.; Dias, J. (2010). “Choques de políticas econômicas e efeitos repercussão entre economias da América Latina e EUA”. Series Working Paper BNDES/ $A N P E C, \mathrm{n}^{\mathrm{o}} 12$, pp.1-43.

Dias, M. H. A.; Dias, J.; Punzo, L. (2012). International Interdependence and Macroeconomic Transmission: Europe and Latin America. In: Beyond the Global Crisis: Structural Adjustments and Regional Integration in Europe and Latin America, London, pp.79-90.

Eurostat. Statistics. Disponível em: < http://ec.europa.eu/eurostat/data/database> Acesso em: ago. 2014.

Frankel, J. A. (1988). “Obstacles to International Macroeconomic Policy Coordination”. Journal of Public Policy, v.8(3/4), pp. 353-374.

IBGE. Contas nacionais trimestrais. Disponível em:

<http://www.ibge.gov.br/home/estatistica/indicadores/pib/pib-vol-val_201401_8. shtm> Acesso em: jun. 2014.

IPEADATA. Estatísticas. Disponível em: <http://www.ipeadata.gov.br/> Acesso em: jun. 2014.

Ministério do Desenvolvimento, Indústria e Comércio Exterior (MDIC). (2013). Balança comercial brasileira: dados consolidados. Disponível em: < http:// www.mdic.gov.br/arquivos/dwnl_1394635352.pdf> Acesso em: mar 2014.

Ministério do Desenvolvimento, Indústria e Comércio Exterior (MDIC). (2014). Intercâmbio comercial brasileiro: países e blocos econômicos. Disponível em: <http://www.desenvolvimento.gov.br/sitio/interna/interna. php?area $=5 \& m e n u=576>$ Acesso em: jun. 2014 . 
FÜHR, J.; DIAS, M. H. A. Transmissão de Política Macroeconômica: o caso de Brasil e Alemanha

Obstfeld, M.; Rogoff, K. (1995). “Exchange Rate Dynamics Redux”. The Journal of Political Economy, v. 103(3), pp. 624-66o.

Oudiz, G.; Sachs, J. (1984). "Macroeconomic Policy Coordination among the Industrial Economies". Brooking Papers on Economic Activity, $\mathrm{n}^{\mathrm{o}} 1$. 


\section{QUADRO 1 - SOLUÇÕES DO MODELO DE CORSETTI E PESENTI (2001)}

\begin{tabular}{|c|c|}
\hline \multicolumn{2}{|c|}{ Soluções do Modelo de Corsetti e Pesenti (2001) } \\
\hline \multicolumn{2}{|c|}{ Determinantes do bem-estar doméstico } \\
\hline (1) $C=a_{1}\left(\bar{M}_{W}\right)^{1 / \rho}$ & Consumo de curto prazo \\
\hline (2) $Y=a_{2}\left(\bar{M}_{R}\right)^{1-\gamma}\left(\bar{M}_{W}\right)^{1 / \rho}+G$ & Produto de curto prazo \\
\hline (3) $\bar{M} / P=a_{3} \bar{M}_{W}$ & Saldos monetários reais no curto prazo \\
\hline (4) $\bar{C}=a_{4}\left(\bar{g}_{W}\right)^{-1 /(1+\rho)}$ & Consumo de longo prazo \\
\hline (5) $\bar{Y}=a_{5}(\bar{g})^{1 / 2}\left(\bar{g}_{W}\right)^{-(1-\rho) /[2(1+\rho)]}$ & Produto de longo prazo \\
\hline (6) $\bar{M} / \bar{P}=a_{6}\left(\bar{g}_{W}\right)^{-\rho /(1+\rho)}$ & Saldos monetários reais no longo prazo \\
\hline \multicolumn{2}{|c|}{ Preços } \\
\hline (7) $1+r=a_{7}\left(\bar{M}_{W}\right)^{-1}\left(\bar{g}_{W}\right)^{-\rho /(1+\rho)}$ & Taxa real de juros de curto prazo \\
\hline (8) $E P_{F}^{*} / P_{H}=a_{8} \bar{M}_{R}$ & Termos de troca de curto prazo \\
\hline (9) $E=\bar{E}=a_{9} \bar{M}_{R}$ & Taxa de câmbio nominal \\
\hline (10) $\bar{E} \bar{P}_{F}^{*} / \bar{P}_{H}=a_{10}\left(\bar{g}_{R}\right)^{-1 / 2}$ & Termos de troca de longo prazo \\
\hline (11) $\bar{P}_{H}=a_{11} \bar{M}\left(\bar{g}_{W}\right)^{-(1-\rho) /[2(1+\rho)]}(\bar{g})^{1 / 2}$ & Preço dos bens domésticos no longo prazo \\
\hline
\end{tabular}

em que o índice R se refere à razão entre as variáveis domésticas e estrangeiras. $\mathrm{O}$ índice $\mathrm{W}$ se refere às médias geométricas das variáveis domésticas e estrangeiras com pesos $\gamma$ e $\gamma-1$. As constantes são definidas como em Corsetti e Pesenti (2001), onde o subscrito o indica nível pré-choque. Variáveis pós-choque são indexadas pela barra superior.

$$
\begin{aligned}
& \alpha_{1}=\gamma\left(\gamma_{w}\right)^{1-\rho / 1+\rho}\left(g_{w o}\right)^{-1 /(1+\rho)}\left(M_{w o}\right)^{1 /(1+\rho)} ; \\
& a_{2}=\gamma^{(1-\rho) / 2}\left(\gamma_{w}\right)^{(1-\rho)^{\wedge} 2 /[2(1+\rho)]}\left(g_{w o}\right)^{-(1-\rho) /[2(1+\rho)]}\left(g_{0}\right)^{-1 / 2}\left(M_{R o}\right)^{-(1-\gamma)}\left(M_{w o}\right)^{-1 / \rho \Phi 1 / 2}\left(\Phi_{w}\right)^{(1-\rho) /} \\
& a_{3}=\chi[(1+\rho) / \rho] \gamma^{\rho}\left(\gamma_{w}\right)^{\rho(1-\rho) /(1+\rho)}\left(g_{w o}\right)^{-\rho(1+\rho)} M^{-1}\left(\Phi_{w}\right)^{\rho /(1+\rho)} ; \\
& a_{4}=\gamma\left(\gamma_{w}\right)^{(1-\rho) /(1+\rho)}\left(\Phi_{w}\right)^{1 /(1+\rho)} ; \\
& a_{5}=\gamma^{(1-\rho) / 2}\left(\gamma_{w}\right)^{(1-\rho)^{\wedge} 2 / 2[(1+\rho)]} \Phi^{1 / 2}\left(\Phi_{w}\right)^{(1-\rho) / 2[(1+\rho)]}
\end{aligned}
$$


$a_{6}=\chi[(1+\delta) / \delta] \gamma^{\rho}\left(\gamma_{w}\right)^{[\rho(1-\rho)] /(1+\rho)}\left(\Phi_{w}\right)^{\rho /(1+\rho)}$

$\alpha_{7}=\beta^{-1}\left(g_{w o}\right)^{\rho /(1+\rho)} M_{w o} ;$

$\alpha_{8}=[\gamma /(1-\gamma)]^{-(1+\rho) / 2}\left(g_{\mathrm{Ro}}\right)^{-1 / 2}\left(M_{\mathrm{Ro}}\right)^{-1} \Phi_{\mathrm{R}}^{1 / 2}$

$\alpha_{9}=\chi^{*} \chi^{-1}[\gamma /(1-\gamma)]^{-\rho}$

$\alpha_{10}=[\gamma /(1-\gamma)]^{-(1+\rho) / 2}\left(\Phi_{\mathrm{R}}\right)^{1 / 2}$

$\alpha_{11}=\left(\alpha_{4}\right)^{\rho} \alpha_{5}\left(\alpha_{6}\right)^{-1} \Phi^{-1}$

Fonte: Corsetti e Pesenti (2001, p. 433).

Anexo

TABELA 1 - TESTES DE RAIZ UNITÁRIA DAS VARIÁVEIS

\begin{tabular}{l|c|c|c|c|c|c|c|c}
\hline \multirow{2}{*}{ VARIÁVEL } & \multicolumn{2}{|c|}{ DF } & \multicolumn{2}{c|}{ PP } & \multicolumn{2}{c|}{ KPSS } & \multicolumn{2}{c}{ DF-GLS } \\
\cline { 2 - 9 } & $\begin{array}{c}\mathrm{t} \\
\text { calculado }\end{array}$ & $\begin{array}{c}\mathrm{t} \\
\text { crítico }\end{array}$ & $\begin{array}{c}\mathrm{t} \\
\text { calculado }\end{array}$ & $\begin{array}{c}\mathrm{t} \\
\text { crítico }\end{array}$ & $\begin{array}{c}\mathrm{t} \\
\text { calculado }\end{array}$ & $\begin{array}{c}\mathrm{t} \\
\text { crítico }\end{array}$ & $\begin{array}{c}\mathrm{t} \\
\text { calculado }\end{array}$ & $\begin{array}{c}\mathrm{t} \\
\text { crítico }\end{array}$ \\
\hline $\operatorname{lng}_{\mathrm{br}}$ & $-4.040^{*}$ & -3.492 & $-4.311^{*}$ & -3.492 & $0.0853^{*}$ & 0.216 & $-5.599^{*}$ & -3.513 \\
$\operatorname{lng}_{\mathrm{w}}$ & $-3.935^{*}$ & -3.492 & $-4.307^{*}$ & -3.492 & $0.0905^{*}$ & 0.216 & $-5.906^{*}$ & -3.513 \\
$\operatorname{lng}_{\mathrm{r}}$ & $-4.112^{*}$ & -3.492 & $-4.339^{*}$ & -3.492 & $0.0902^{*}$ & 0.216 & $-5.505^{*}$ & -3.513 \\
dlnpib_br & $-9.418^{*}$ & -3.492 & $-9.330^{*}$ & -3.492 & $0.175^{*}$ & 0.221 & $-6.210^{*}$ & -3.514 \\
dlncambio & $-8.180^{*}$ & -3.492 & $-8.215^{*}$ & -3.492 & $0.16^{*}$ & 0.224 & $-6.587^{*}$ & -3.514 \\
\hline
\end{tabular}

${ }^{*}$ Significante a $1 \%$;

Fonte: Elaboração própria a partir dos resultados do Stata. 
GRÁFICO 3- DISTRIBUIÇÃO DOS RESÍDUOS DO PRODUTO E DOS TERMOS DE TROCA
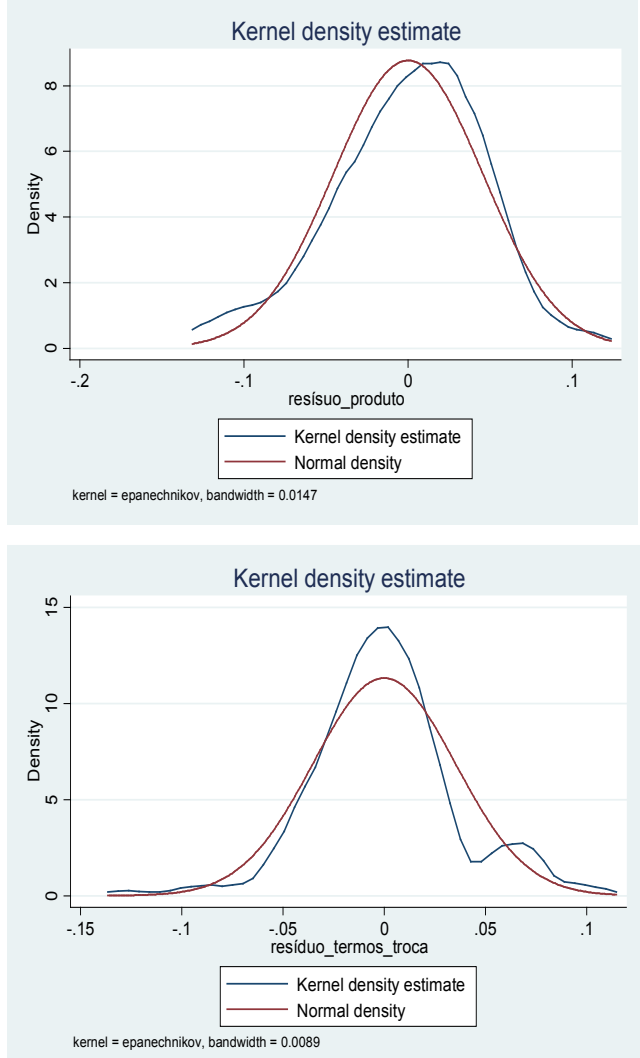

Fonte: Elaboração própria a partir dos resultados da pesquisa no Stata.

TABELA 2 - TESTES DE ESTACIONARIDADE DOS RESÍDUOS DO PRODUTO E DOS TERMOS DE TROCA RESPECTIVAMENTE

\begin{tabular}{|l|rr||l|rr|}
\hline TESTE & $\mathbf{t}_{\text {calculado }}$ & $\mathbf{t}_{\text {crítico }} \mathbf{1 \%}$ & TESTE & $\mathbf{t}_{\text {calculado }}$ & $\mathbf{t}_{\text {crítico }} \mathbf{1 \%}$ \\
\hline DF & -11.679 & -3.494 & DF & -11.890 & -3.494 \\
PP & -11.679 & -3.494 & PP & -11.888 & -3.494 \\
KPSS & 0.0825 & 0.216 & KPSS & 0.041 & 0.216 \\
DF-GLS & -8.037 & -3.520 & DF-GLS & -9.048 & -3.524 \\
\hline
\end{tabular}

Fonte: Elaboração própria a partir dos resultados da pesquisa no Stata. 